\title{
ÉTUDE SUR LES PIVOTS A GRAINS PARALLĖLES
}

par

\section{P. BOUSSUGES}

\author{
lngénieur E. I. H.
}

Professeur à l'école des Ingénieurs hydrauliciens de Grenoble

\section{S. CASACCI}

ingénieur $A$, et $M$

Ingénieur aux Ateliers N. B P. P.
En 1883 BEAUCHAMP-TOWER s'aperçut qu'un tourillon tournant dans un coussinet abondamment graissé était capable de supporter d'assez fortes charges avec un faible coefficient de frottement. II supposa que cette réduc tion de frottement était due ò l'écoulement d'un mince film d'huile entre les deux surfaces frottantes, en mouvement l'une par rapport d̀ l'autre.

Ce phénomène, très important du point de vue technique, fut étudié pour la première fois par OSBORNE REYNOLDS( $\mathrm{r}$ ). Ce physicien prouva analytiquement qu'il suffisait que les deux surfaces frottantes puissent former un angle pour que le graissage hydro-dynamique s'établisse, c'est-à-dire pour que les deux surfaces soient séparées par un film d'huile appelé « coin d'huile». En s'inspirant de cette théorie, MICHELL $(*)$ inventa son ingénieuse butée d̀ patins oscillants qui est encore largement utilisée dans la construction mécanique.

L'étude classique du graissage hydrodynamique par « coin d'huile » fut ensuite complétée par BOSWALL. Dans son remarquable ouvrage (3) on trouve exposée une théorie complète de la pivoterie MICHELL à patins oscillants qui tient compte, dans une certaine mesure, de la variation de la viscosité de l'huile avec la température, grâce à l'introduction d'une viscosité moyenne dans les équations du phénomène.

Les théories de OSBORNE REYNOLDS, MICHELL et BOSWALL n'étaient pas dans toutes leurs conséquences en accord avec les faits expérimentaux. Elles n'expliquaient pas, en effet, la capacité de charge des pivoteries à grains parallèles. Certains appareils, conçus sur ce principe (crapaudines, glissières de machines outils, etc.), fonctionnaient cependant avec de

(1) Osborne Reynolds "The Theorie of Lubrication " Phil Trans Royal Society 1886.

(2) Michell, A.G.M. « Lubrciation of .Plane Surfaces » Zeit. für Math. und Phy. 1905.

(3) Boswall "The theorie of film lubrication " 1928 faibles coefficients de frottement. On admit alors que la mince pellicule d'huile séparant les sur. faces frottantes était due aux qualités « onc-

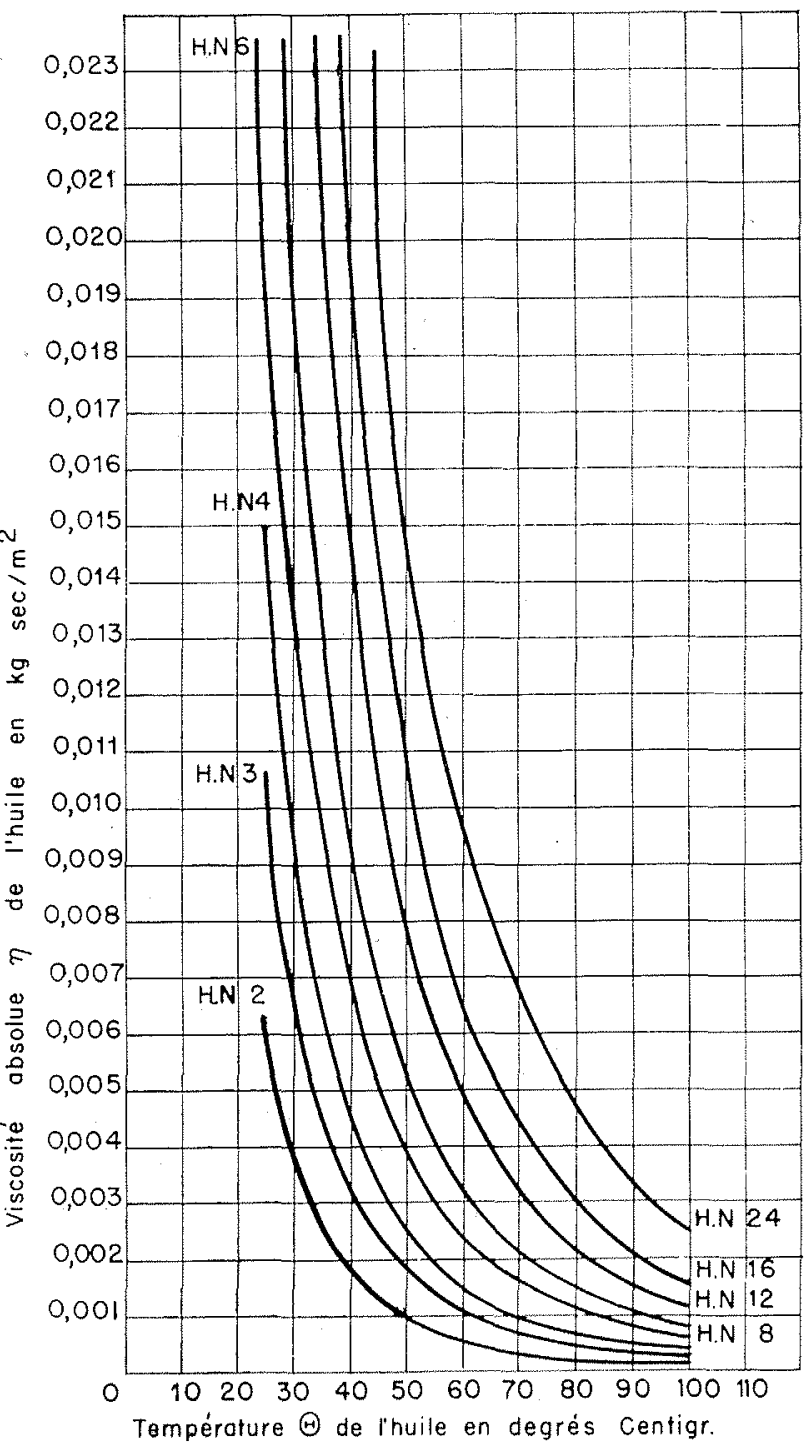

Fig. 1 
tueuses 》du lubrifiant et l'on caractérisa ce phénomène en le nommant régime « onctueux » pour le différencier du régime hydrodynamique.

Le problème du fonctionnement de ces appareils suscita diverses interprétations, mais ce $n^{\prime}$ est qu'après les essais de $M$. FOGG que $M$. CAMERON et Mrs. WOOD l'expliquèrent analytiquement en 1942. C'est, à notre connaissance, la première théorie du film plan reposant sur la variation de la masse spécifique du lubrifiant avec la température. Ces auteurs ont envisagé le cas du patin plan de largeur infinie, la viscosité de I'huile restant constante. Tout récemment, M. SHAW a publié dans I'A.S.M.E. de mai 1947, une étude simplifiée reposant sur la même propriété physique de l'huile. Cela lui o permis de comparer la capacité de charge des pivoteries à patins oscillants et à patins plans.

La théorie précédente est basée sur la variation de la masse spécifique de I'huile avec la température. Cette variation étant en général assez faible, toute charge notable nécessite une élévation de température importante entrainant une forte diminution de la viscosité (Cf. fig. 1). On conçoit donc l'intérêt de tenir compte de la variation de viscosité dans le cas d'un patin de glissière de largeur finie et d'étendre la méthode au cas des pivoteries, questions qui n'ont pas été traitées par les auteurs cités précédemment. C'est ce que nous nous proposons d'examiner dans l'étude suivante.

\section{NOTATIONS}

$$
\begin{aligned}
& \text { L } \quad=\text { longueur du patin. } \\
& \text { I }=\text { largeur du patin. } \\
& \mathrm{h}=\quad=\text { hauteur du film (constant par hy- }
\end{aligned}
$$

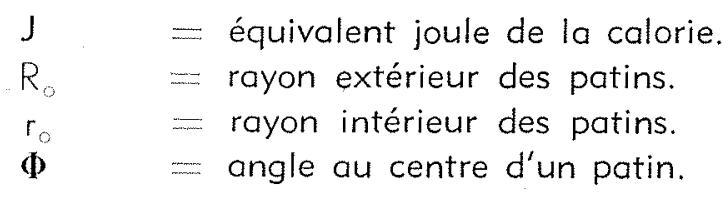

\section{CAS DU PATIN DE GLISSIERE}

\section{Hypothèses admises :}

La variation de température est linéaire le long du patin.

$$
\Delta t=\lambda x
$$

La température ne dépend pas de la variable y.

Les variations de la masse spécifique et de la viscosité de I'huile avec la température peuvent être représentées avec une très bonne approximation par les formules suivantes:

$$
\begin{aligned}
& \rho=\rho_{0} e^{-\alpha \Delta t} \\
& \mu=\mu_{0} e^{-\beta \Delta t}
\end{aligned}
$$

Ces relations sont valables pour les élévations de températures qui se produisent sous les parties et qui dépassent rarement $30^{\circ}$.

Le coefficient $\alpha$ est sensiblement le même pour toutes les huiles et a pour valeur $7 \times 10^{-1}$.

Le coefficient $\beta$ est une constante physique fonction de l'index de viscosité de l'huile.

Rappelons les équations générales de l'écoulement des fluides visqueux.

$$
\left[\begin{array}{l}
\frac{\partial p}{\partial x}=\mu \frac{\partial^{2} u}{\partial z^{2}} \\
\frac{\partial p}{\partial y}=\mu \frac{\partial^{2} v}{\partial z^{2}}
\end{array}\right.
$$

Supposant $p$ indépendant de $z$, on en tire:

$$
\left[\begin{array}{l}
u=\frac{1}{2 \mu} \cdot \frac{\partial p}{\partial x}\left(z^{2}-z h\right)+\frac{u z}{h} \\
v=\frac{1}{2 \mu} \cdot \frac{\partial_{p}}{\partial y}\left(z^{2}-z h\right)
\end{array}\right.
$$

En intégrant ces relations par rapport à $z$, nous obtiendrons les éléments de débit suivant les axes $O x$ et $O y$, soit :

$$
\begin{aligned}
& e^{2 \gamma \tau}=\sum_{m=1}^{m_{z} \infty} K_{m} \sin m \tau \\
& \text { avec } K_{m}=\frac{2 m}{\pi} \cdot \frac{1-e^{2 \gamma \pi}}{m^{2}+4 \gamma^{2}}
\end{aligned}
$$

En adjoignant à ces deux égalités l'équation de continuité : $\frac{\partial}{\partial x} \rho q_{x}+\frac{\partial}{\partial y} \rho q_{y}=0$ 

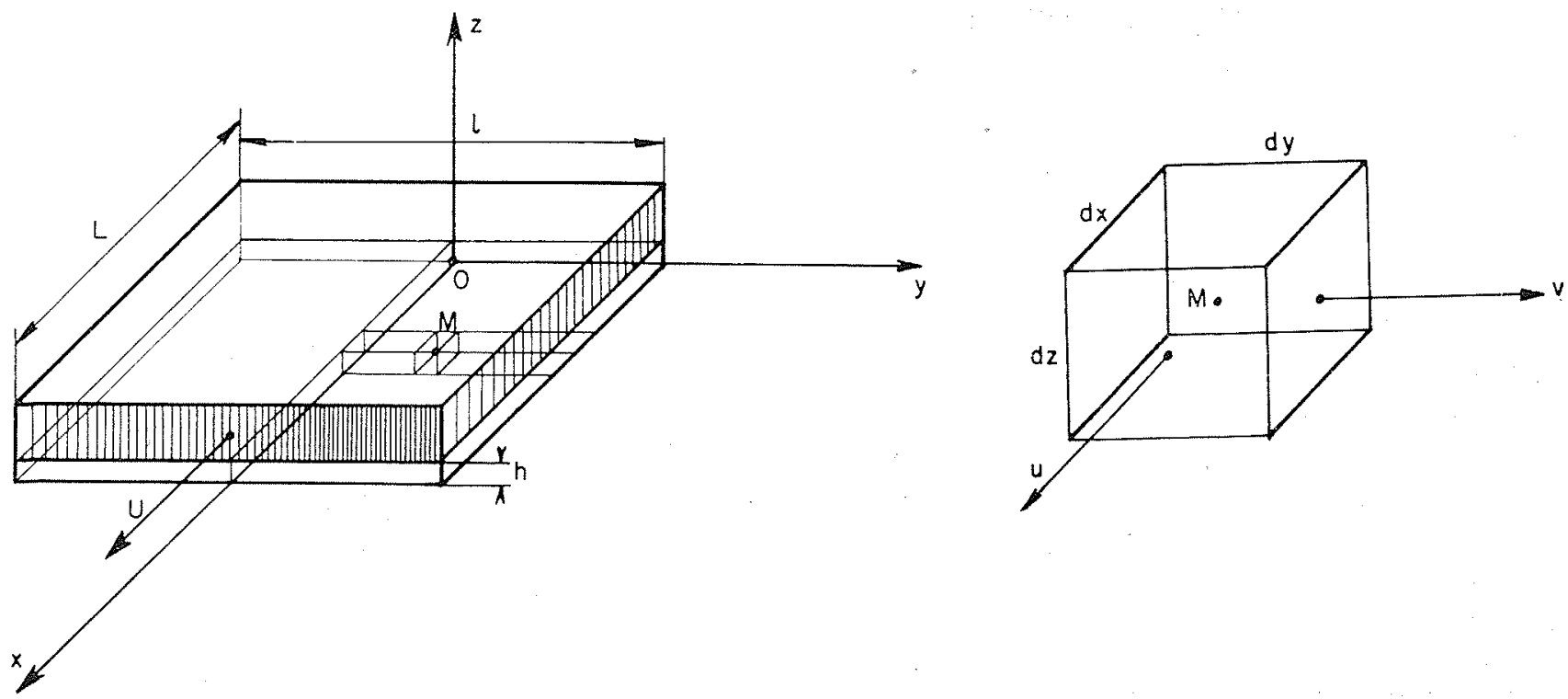

Fig. 2

nous sommes conduits à l'équation aux dérivées partielles:

$$
\frac{\partial^{2} p}{\partial x^{2}}+\frac{\partial_{p}^{2}}{\partial y^{2}}+\lambda(\beta-\alpha) \frac{\partial p}{\partial x}+\frac{6 U \alpha \lambda \mu_{\alpha}, e^{-\beta \lambda x}}{h^{2}}=0
$$

qui résout le problème lorsque $\lambda$ est connu.

11 est facile de montrer que la fonction $p(x, y)$ admet un maximum positif, ce qui prouve l'existence du film plan.

Sachant que dans l'intervalle $-\frac{1}{2}<y<+\frac{1}{2}$

$$
\frac{\pi}{4}=\sum_{n=0}^{n=\infty} \frac{1}{2 n+1} \sin (2 n+1) \frac{\pi}{2}\left(y+\frac{1}{2}\right)
$$

et posant

$$
K=\frac{24 \alpha \lambda \mu_{0}}{\pi h^{2}} \cdot u
$$

la solution de l'équation (il) peut être mise sous la forme suivante:

$$
p=K \sum_{n=0}^{n=\infty} \frac{p_{n}(x)}{2 n+9} \sin \cdot(2 n+1) \frac{\pi}{1}\left(y+\frac{1}{2}\right)
$$

Les fonctions $p_{\mathrm{n}}(\mathrm{x})$ satisfaisant d̀ l'équation :

$\frac{d^{2} p_{n}(x)}{d x^{2}}+\lambda(\beta-\alpha) \frac{d p_{n}(x)}{d x}-\frac{\pi^{2}(2 n+1)^{2}}{t^{2}} p_{n}(x)+e^{-\beta \lambda x}=0$

dont la solution générale est :

$$
p_{n}(x)=M_{n} e^{a_{n}^{\prime} x}+N_{n} e^{a_{n}^{n} x}+k_{n} e^{-\beta \lambda x}
$$

les coefficients $M_{1 k}$ et $N_{11}$ sont déterminés par les conditions initiales:

$$
P_{n}(0)=0 \text { et } P_{n}(L)=0
$$

$a^{\prime}{ }_{1}$ et $a^{\prime \prime}{ }_{n}$ sont les racines de l'équation caractéristique de (15)
La charge totale est donnée par:

$$
\begin{gathered}
P=\int_{0}^{L} \int_{-\frac{l}{2}}^{+\frac{l}{2}} p d x d y \text {, soif } \\
P=\frac{2 l}{\pi} \cdot K \sum_{n=0}^{n=\infty} \frac{1}{(2 n+1)^{2}}\left[\frac{M_{n}}{a_{n}^{\prime}} e^{a_{n}^{1} x}+\frac{N n}{a_{n}^{4}} e^{a_{n}^{\prime \prime} x}-\frac{k_{n}}{\beta \lambda} e^{-\beta \lambda x}\right]_{0}^{L}
\end{gathered}
$$

ou encore:

$$
\Phi=\frac{2 l}{\pi} \cdot K \sum_{n=0}^{n_{n} \infty} \frac{1}{(2 n+1)^{2}}\left[\frac{M_{n}}{a_{n}^{\prime}}\left(e^{a_{n}^{n} L}-1\right)+\frac{N_{n}}{a_{n}^{n}}\left(e^{a_{n}^{n} L}-1\right)+\frac{k_{n}}{\beta \lambda}\left(1-e^{-\beta \lambda L}\right)\right] \text { (18) }
$$

\section{Puissance absorbée par le frottement :}

L'effort tangentiel s'exerçont sur un élément de surface du patin mobile est donné par:

$$
T_{h}=-\mu\left(\frac{\partial u}{\partial z}\right)_{z=h}
$$

soit : $\quad T_{h}=-\frac{h}{2} \frac{\partial p}{\partial x}+\frac{u}{h} \cdot \mu$

La force totale de frottement est donc:

$$
F=\int_{0}^{L} \int_{-\frac{1}{2}}^{+\frac{1}{2}} T_{h} d x d y=\frac{u l \mu_{0}}{h \beta \lambda}\left[1-e^{-\beta \lambda L}\right]
$$

ce qui donne pour la puissance absorbée :

$$
w=F \cdot U=\frac{u^{2} 1 \mu_{0}}{h \beta \lambda}\left[1-e^{-\beta \lambda L}\right]
$$

Nous supposerons que la puissance absorbée se dissipe entièrement en échauffant le film d'huile.

\section{Calcul de l'élévation de température:}

Le débit moyen d'huile du film est approxi- 
mativement représenté par l'équation :

$$
0=\frac{u h}{2} 1
$$

Le bilan calorifique est alors:

$$
\frac{W}{J}=\rho_{0} c Q \Delta t
$$

\section{Formules et résultats pratiques :}

Reprenons la formule générale donnant la charge et ne conservons que les premiers termes. nous obtenons alors:

$$
P=\frac{21}{\pi} \cdot K\left[\frac{M_{0}}{\sigma_{0}^{\prime}}\left(e^{a_{0}^{\prime} L}-1\right)+\frac{N_{0}}{a_{0}^{\prime}}\left(e^{a_{0}^{\prime \prime} L}-1\right)+\frac{k_{Q}}{\beta \lambda}\left(1-e^{-\beta \lambda L}\right)\right]
$$

Les deux premiers termes de la parenthèse sont négatifs et peuvent être négligés vis-à-vis du troisième terme.

La relation donnant la.charge devient alors:

$$
P=\frac{2 l}{\pi} \cdot k\left[\frac{k_{0}}{\beta \lambda}\left(1-e^{-\beta \lambda L}\right)\right]
$$

$$
\text { avec } K_{13}=\frac{1}{\frac{\pi^{2}}{1^{2}}-\alpha \beta \lambda^{2}}
$$

le terme $\alpha \beta \lambda^{*}$ étant négligeable devant $\frac{\pi^{*}}{1 *}$ nous aurons done :

$$
P=\frac{1}{h^{2}} \cdot \frac{48}{\pi^{4}} \cdot \frac{a}{\beta} \cdot \mu_{0} \cdot U \cdot l^{3}\left(1-e^{-\beta \lambda L}\right)
$$

en introduisant la pression spécifique

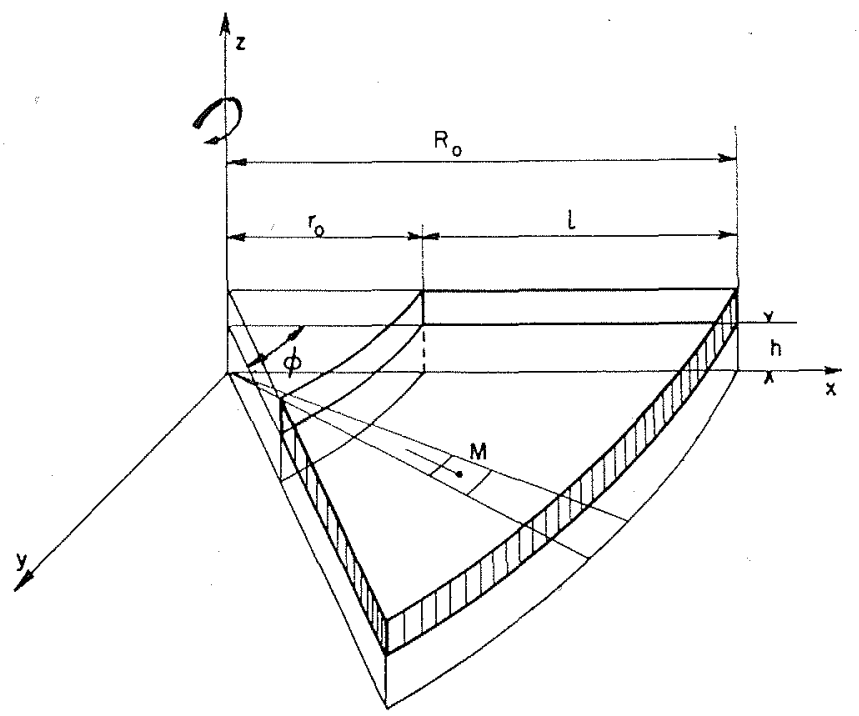

$P_{s}=\frac{P}{1>1}$ l'épaisseur du film est donnée par la relation:

$$
h^{2}=\frac{48}{\pi^{4}} \cdot \frac{a}{\beta} \cdot \frac{\mu_{0}}{p_{s}} \cdot U \cdot 1^{2}\left(1_{-} \mathrm{e}^{-\beta \lambda L}\right)
$$

soit :

$$
\frac{h}{l}=\sqrt{\frac{48}{\pi^{4}} \frac{a}{\beta}\left(1-e^{-\beta \lambda L}\right)} \cdot \sqrt{\frac{\mu_{0} u}{\rho_{s}}}
$$

qui caractérise l'élévation de température à l'aide de la formule: $\quad \lambda=\frac{2}{l} \sqrt{\frac{p_{s}}{J \rho c \alpha}}$

Remarque 1.: Dans le cas de la glissière, io répartition des pressions est évidemment symétrique par rapport à l'axe $O x$. On peut alors facilement arriver aux mêmes résultats en supposant que la loi de répartition de la pression suivant la direction oy est une parabole. La pression est alors représentée par la relation:

$$
p=p_{0}(x)\left[1-\left(\frac{2 y}{l}\right)^{2}\right]
$$

$p_{0}(x)$ satisfaisant à l'équation différentielle: $\frac{d^{2} p_{0}}{d x^{2}}+\lambda(\beta-a) \frac{d p_{0}}{d x}-\frac{6 p_{0}}{l^{2}}+\frac{6 U \alpha \lambda \mu_{0}}{h^{2}} e^{-\beta \lambda x}=0$ qui admet pour solution :

$P_{0}=M e^{-a_{0} x}+N e^{-a_{2} x}+\frac{3}{4} \frac{u \alpha \lambda t^{2} \mu_{0} e^{-\beta \lambda x}}{n^{2}}=0$

les constantes $M$ et $N$ étant déterminées par les conditions aux limites

$$
P_{0}(0)=0 \text { et } P_{0}(L)=0
$$

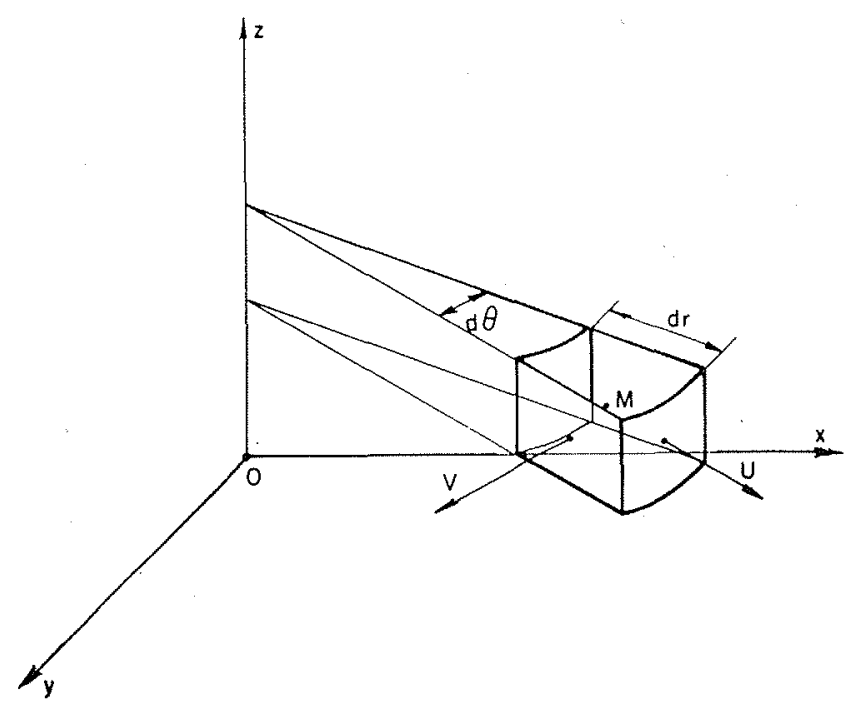

Fig. 3 
En faisant des approximations analogues d̀ celles de la méthode précédente, approximations justifiées par la faible valeur des coefficients $\alpha$ et $\beta$, on arrive aux relations suivantes donnant l'épaisseur du film d'huile et l'élévation de température :

$$
\frac{n}{l}=\sqrt{\frac{\alpha}{2 \beta L} \cdot\left(1-e^{-\beta \lambda L}\right)} \cdot \sqrt{\frac{\mu_{0} U}{P_{s}}}
$$

et

$$
i=\frac{2}{i} \sqrt{\frac{p_{s}}{j \rho_{0} c a}}
$$

Remarque 11: L'examen de la formule (34) montre que l'épaisseur du film d'huile dépend de 4 facteurs :
1) $\sqrt{\frac{\mu_{0} U}{p_{s}}}$
facteur classique que l'on re- trouve dans tous les problèmes de graissage.
2) $\left(1-e^{-\beta \lambda L}\right)$
caractérise l'influence de l'élé- vation de température sur $h$. On voit en effet que pour des températures importantes, ce facteur tend vers 1. Nous I'ap- pellerons «facteur calorifique ».
3) $\alpha$ facteur sensiblement le même pour toutes les huiles.
4) $\quad \beta L \quad$ coefficient montrant l'influence de la variation de la viscosité avec la température. Les huiles d'index de viscosité élevé amé- liorent donc l'épaisseur du film.

\section{CAS D'UNE PIVOTERIE}

En coordonnées cylindriques, les équations du mouvement et de continuité deviennent :

(Cf. fig. $n^{\circ} 3$ )

$$
\begin{aligned}
& q_{\theta}=-\frac{1}{\mu r} \frac{\partial p}{\partial \theta} \frac{h^{3}}{12}+\frac{\omega r h}{2} \\
& q_{r}=-\frac{1}{\mu} \frac{\partial p}{\partial r} \frac{h^{3}}{12} \\
& \frac{\partial}{\partial r} \rho r q_{r}+\frac{\partial}{\partial \theta} \rho q_{\theta}=0
\end{aligned}
$$

et conduisent à l'équation aux dérivées partielles: $\frac{\partial^{2} p}{\partial \theta^{2}}+r \frac{\partial}{\partial r}\left(r \frac{\partial p}{\partial r}\right)+\lambda(\beta-\alpha) \frac{\partial p}{\partial \theta}+\frac{6 \alpha \lambda \omega r^{2} \mu_{0}}{h^{2}} e^{-\beta \lambda \theta}=0$ Posons: $r=$ ro $\sigma^{\gamma \tau}$ avec ro $<r<$ Ro et $O<\tau<\pi$ ce qui donne pour valeur de $\gamma$ :

$$
\gamma=\frac{1}{\pi} \log _{\mathrm{n}} \frac{R}{r_{0}}
$$

L'équation $\left(4^{\prime}\right)$ devient alors:

$\frac{\delta^{2} p}{\partial \theta^{2}}+\frac{1}{\gamma^{2}} \frac{\partial^{2} p}{\partial \tau^{2}}+\lambda(\beta-a) \frac{\partial p}{\partial \theta}+\frac{6 a \lambda \omega r_{Q}^{2} \mu_{\theta}}{h^{2}} e^{2 \gamma \tau} e^{-\beta \lambda \theta}=0$

en posant :

$$
\frac{6 \alpha \lambda \omega r_{0}^{2} \mu_{0}}{n^{2}}=A
$$

Considérons la fonction $F(\tau)$ définie par la série de FOURIER :

$$
e^{2 \lambda \tau} F(\tau)=\sum_{m=1}^{m=\infty} k_{m} \sin m \tau
$$

Un calcul simple montre que :

$$
F(\tau)=\left[\begin{array}{ll}
1 & \text { pour } 0<\tau<\pi \\
-1 & \text { pour }-\pi<\tau<0
\end{array}\right.
$$

Dans la suite, nous utiliserons $F(\tau)$ que dans l'intervalle $\mathrm{O}<\tau<\pi$

Nous pouvons donc écrire:

$$
\left[\begin{array}{l}
q_{x}=-\frac{h^{3}}{12 \mu} \cdot \frac{\partial p}{\partial x}+\frac{v h}{2} \\
q_{y}=-\frac{h^{3}}{12 \mu} \cdot \frac{\partial p}{\partial y}
\end{array}\right.
$$

pour $m$ pair.

$$
\text { et } \quad k_{m}=\frac{2 m}{\pi} \quad \frac{1+e^{2 \gamma \pi}}{m^{2}+4 \gamma^{2}} \quad \text { (10') }
$$

pour $m$ impair.

La solution de cette équation est :

$$
P=A \sum_{m=1}^{m=\infty} F_{m}(\theta) K_{m} \sin m \tau \quad \text { (II) }
$$

$\mathrm{Fm}(A)$ satisfaisant à l'équation :

$\frac{d^{2} F_{m}}{d \theta^{2}}+\lambda(\beta-\alpha) \frac{d F_{m}}{d \theta}-\frac{m^{2}}{\gamma^{2}} F_{m}+e^{-\beta \lambda \theta}=0$

d'où l'on tire :

$$
F_{m}=M_{m} e^{a_{m}^{\prime} \theta}+N_{m} e^{a_{m}^{\prime \prime} \theta}+k_{m} e^{-\beta \lambda \theta}
$$

$a^{\prime}{ }_{\text {In }}$ et $a^{\prime \prime}{ }_{\text {m }}$ étant les racines de l'équation caractéristique et $k_{m}$ une constante ayant pour

$$
\text { valeur: } \quad k=\frac{1}{\left(\frac{m}{y}\right)^{2}-\alpha \beta \lambda^{2}}
$$

Les constantes $M_{m}$ et $N_{\text {m }}$ sont déterminées par les conditions aux limites:

$$
F_{m}(0)=0 \text { et } F_{m}(\Phi)=0
$$

La charge totale est alors donnée par:

$$
P=\int_{r_{0}}^{R_{0}} \int_{0}^{\Phi} p r d \theta d r=A r_{0}^{2} D
$$


avec :

$D=\frac{\pi \gamma}{2} \sum_{m=1}^{m=\infty} K_{m}^{2}\left[\frac{M_{m}}{a_{m}^{1}}\left(e^{a_{m}^{\prime} \Phi}-1\right)+\frac{N_{m}}{a_{m}^{\prime \prime}}\left(e^{a_{m}^{\prime \prime} \Phi}-1\right)+\frac{k_{m}}{\beta \lambda}\left(1-e^{-\beta \lambda \Phi}\right)\right]$

L'épaisseur du film d'huile s'obtient par la formule :

$$
h=r_{0} \sqrt{6 \alpha \lambda D} \sqrt{\frac{\omega \mu_{0}}{p}}
$$

soit en introduisant la pression spécifique (ps) et en posant :

$$
\begin{aligned}
& s=B \Phi r_{0}^{2} \text { avec } B=\frac{1}{2}\left[\left(1+\frac{1}{r_{0}}\right)^{2}-1\right] \\
& h=r_{0} \sqrt{\frac{6 \alpha \lambda D}{B \Phi}} \sqrt{\frac{\omega \mu_{0}}{p_{s}}}
\end{aligned}
$$

\section{Calcul de la puissance absorbée :}

Le couple de frottement est donné par:

$$
\begin{aligned}
G & =\int_{r_{0}}^{r_{\sigma^{+}}^{8}} \int_{0}^{\Phi} T_{h} r^{2} d \theta d r \\
& =\frac{\omega \mu_{0}}{4 h \beta \lambda}\left(R_{0}^{4}-r_{0}^{4}\right)\left(1-e^{-\beta \lambda \Phi}\right)
\end{aligned}
$$

et partant la puissance absorbée par :

$$
w=c \omega=\frac{\omega^{2} \mu_{0}}{4 h \beta \lambda}\left(R_{0}^{4}-r_{0}^{4}\right)\left(1-e^{-\beta \lambda \Phi}\right)
$$

\section{Calcul de l'élévation de température :}

Le débit moyen du film d'huile est de même approximativement représenté par :

$$
\begin{aligned}
& Q=\frac{h l}{2} \int_{r_{0}}^{R_{0}} \omega r d r=\frac{h l}{4}\left(R_{0}^{2}-r_{0}^{2}\right) \omega \\
& \frac{W}{J}=P_{0} \cdot c \cdot Q \cdot \Delta t
\end{aligned}
$$

(20")

\section{Formules et résultats pratiques :}

Le terme $D$ qui entre dans la formule permettant de calculer l'épaisseur du film d'huile peut être simplifié considérablement pour les mêmes raisons que nous avons exprimées précédemment.

11. devient alors:

$$
D=\frac{\pi \gamma}{2} \quad \frac{1-e^{-\beta \lambda \Phi}}{\beta \lambda} \quad k_{1}^{2} k_{1}
$$

avec $k_{1}=\gamma^{\prime \prime}$

$$
\text { d'où : } \quad \mathrm{D}=\frac{\pi \gamma^{3}}{2} \cdot \frac{1-e^{-\beta \lambda \Phi}}{\beta \lambda} \cdot k_{1}^{2}
$$

L'épaisseur du film d'huile s'exprime alors par:

$$
h=3,1 \cdot \gamma \cdot k_{1} \cdot r_{0} \sqrt{\frac{\alpha \gamma\left(1-e^{-\beta \lambda \Phi)}\right.}{B \cdot \beta \cdot \Phi}} \sqrt{\frac{\omega \mu_{0}}{p_{s}}}
$$

Le coefficient $\lambda$ est donné par :

$$
\lambda^{2}=\frac{R_{0}^{4}-r_{0}^{4}}{r_{0}^{4}} \cdot \frac{p_{s}}{3 \pi j \gamma^{3} k_{1}^{2} a c \rho_{0}}
$$

ou bien :

$$
\lambda=\sqrt{\frac{R_{0}^{4}-r_{0}^{4}}{r_{0}^{4}} \cdot \frac{1}{3 \pi \gamma^{3} k_{1}^{2}}} \sqrt{\frac{p_{s}}{1 P_{0} c \alpha}}
$$

\section{ESSAIS ET APPLICATIONS NUMERIQUES}

Des essais effectués sur un gros tour vertical, C.W. BERTHIEZ nous ont permis de vérifier les calculs précédents (cf. fig. $n^{\circ} 4$ ). L'épaisseur du film d'huile a été mesurée mécaniquement ò l'aide de comparateurs à cadran. Les touches de ces appareils frottaient sur des plaques rectifiées, fixées sur la périphérie du plateau de $4 \mathrm{~m}$. Ces plaques étaient suffisamment longues et munies de rampes d'entrée afin de faciliter la mesure qui était vérifiée au moyen d'un comparateur placé au centre du plateau (cf. fig. $n^{\circ} 5$ ).

L'échauffement inégal des parties tournantes
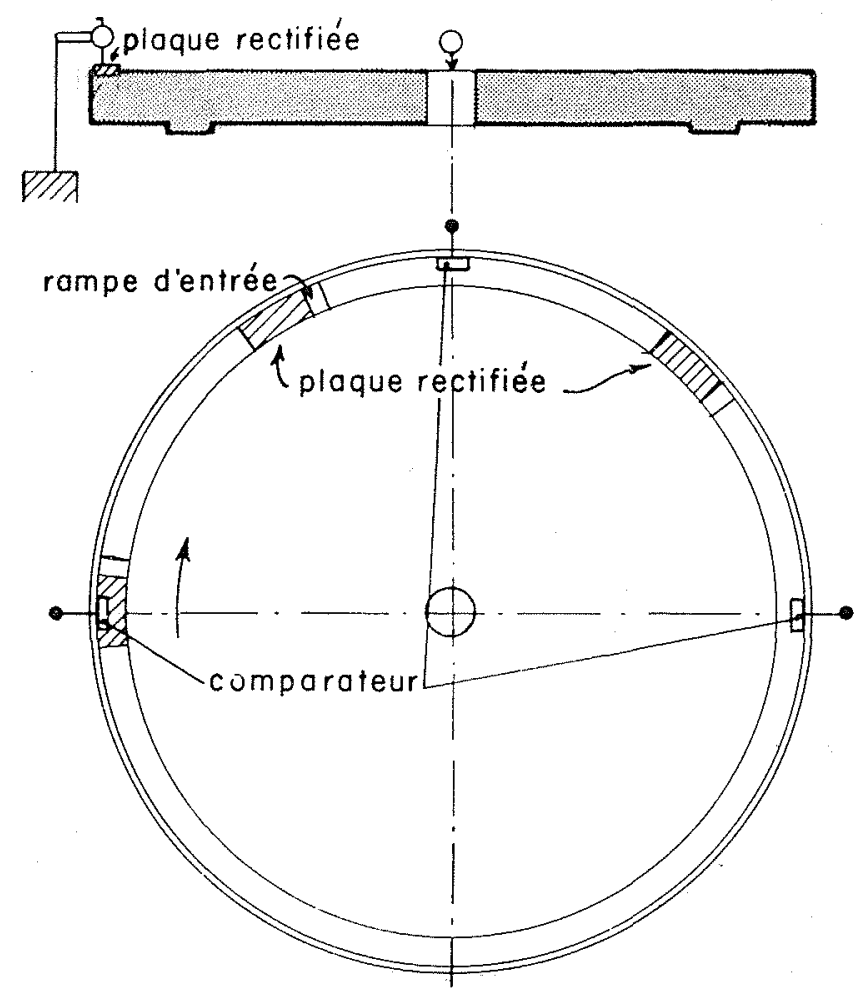

Fig. 5 


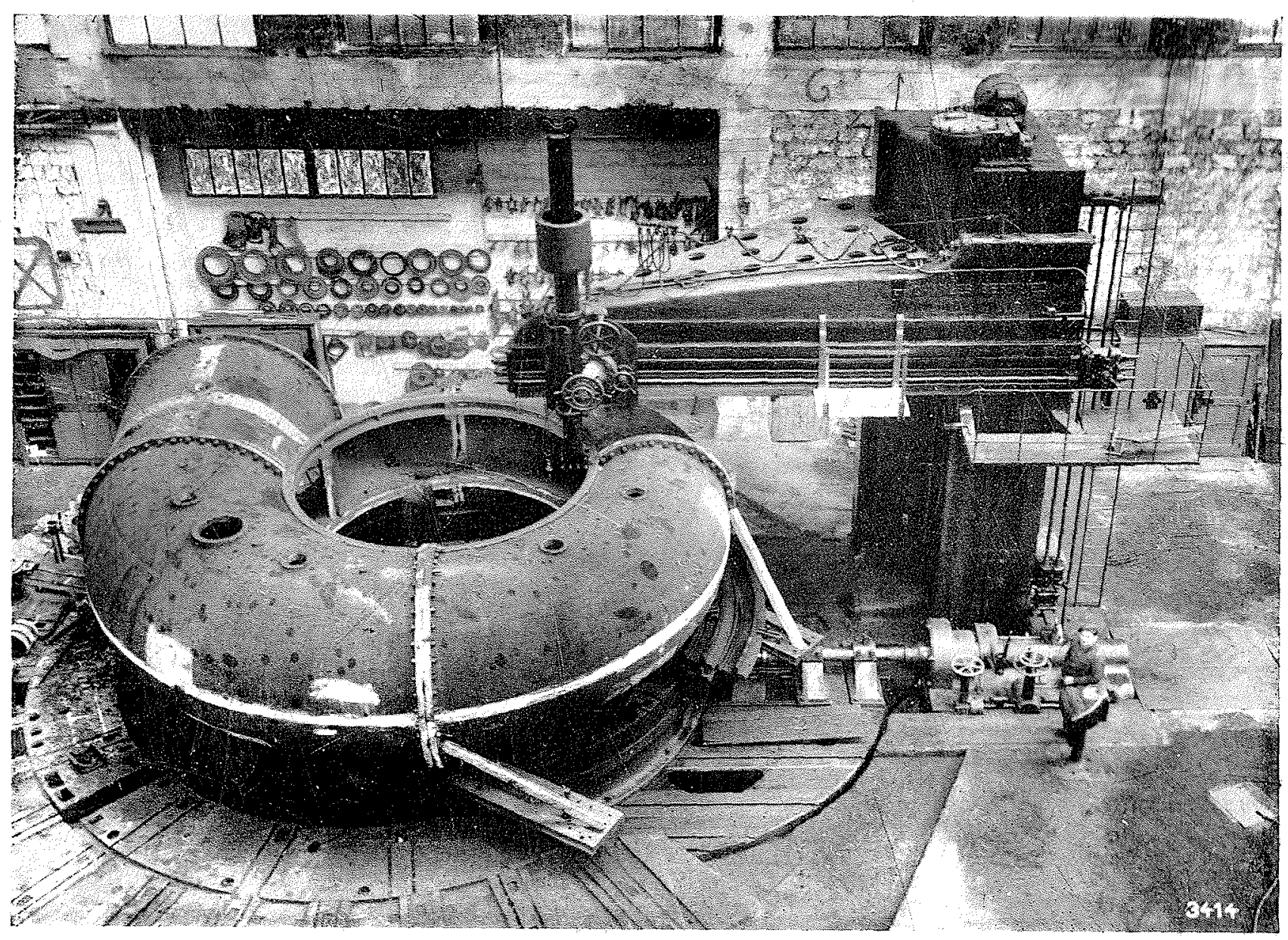

Fig. 4

pouvant provoquer des déformations importantes susceptibles de fausser entièrement les mesures, il fut alors nécessaire d'opérer très rapidement. La température ambiante, sensiblement identique à celle du bac à huile, resta constante et l'on évita un échauffement généralisé des glissières.

Le plateau intérieur du tour reposait sur des glissières de dimensions suivantes (fig. 5):

a) rayon extérieur $1600 \mathrm{~mm}$.

b) rayon intérieur $1400 \mathrm{~mm}$.

c) angle du patin 0,2 radian

L'huile utilisée pendant les essais avait comme caractéristiques

Viscosité à $15^{\circ} \mathrm{C} \ldots . . . . .8$ 80 Engler

Viscosité à $30^{\circ} \mathrm{C} \ldots \ldots \ldots 27^{\circ}$ Engler

Viscosité à $50^{\circ} \mathrm{C} \ldots \ldots \ldots \ldots . . .68^{\circ}$ Engler

L'index de viscosité donné par l'abaque de M. GROFF est de : 50.

Les essais furent effectués avec le plateau non chargé, la pression spécifique supportée par des glissières était d'environ $600 \mathrm{grs} / \mathrm{cm}^{2}$, la température sensiblement voisine du $10^{\circ} \mathrm{C}$.

La figure n: 7 montre la courbe expérimentale obtenue, l'épaisseur du film est explicitée en fonction de la vitesse de rotation du pivot.

Evaluons la valeur de $h$ au moyen des résul. tats analytiques exposés précédemment.

Les formules $19^{\prime}$ et $20^{\prime}$ nous permettent de calculer la fonction $h=f\left({ }_{(0)}\right)$.

Les paramètres contenus dans ces deux équations ont dans le cas présent, les valeurs suivantes:

Ro $=160 \mathrm{~cm}$

ro $=140 \mathrm{~cm}$.

ps $=600 \mathrm{~g}=6 \times 10^{\circ}$ dynes $/ \mathrm{cm}^{2}$.

$\alpha=7 \times 10$.

$B=7 \times 10$

$\mathrm{C}=0,5$ (chaleur spécifique)

$0, \quad=0,9$ (masse spécifique).

$K_{1}=1,47$.

$v=-4.4 \times 10^{-2}$

(1) $=0,2$ radian.

u. $=10$ poises

$\mathrm{B}=0,15$

$\mathrm{J}=4,18 \times 10^{7} \mathrm{erg}$. 
$\lambda$ colculé à l'aide de $\left(20^{\prime}\right)$

$$
\lambda=200
$$

ct: qui correspond à une élévation de température le long du patin de

$$
\lambda \Phi=\Delta t=40^{\circ} \mathrm{C} \text {. }
$$

L.'équation $\left(19^{\prime}\right)$ donne $h=f(n)$

$n$ étant la vitesse de rotation en tours minutes et $h$ l'épaisseur du film en $0,01 \mathrm{de} \mathrm{m} / \mathrm{m}$.

On a:

$$
h=4,5 \sqrt{n}
$$

La figure $n^{\circ} 7$ permet de comparer la couribe de $h(n)$ ainsi calculée et les points expérimentaux

La difficulté d'apprécier les valeurs exactes de la température à l'entrée de chaque glissière suffit à expliquer les écarts existant entre les deux courbes. Ces écarts n'excèdent pas d'ailleurs 2\%:00 de $\mathrm{mm}$.

\section{CONCLUSION}

La théorie précédente en accord avec l'expérience, permet de conclure d̀ l'existence d'un film plan en régime hydrodynamique.

La capacité de charge de ce film était, jus. qu'à ces dernières années, attribuée aux qualités "onctueuses » des huiles: qualités fort mal connues. On conçoit l'intérêt d'expliquer le phénomène en $n^{\prime}$ introduisant dans la mise en équation que des constantes physiques bien définies: viscosité, index de viscosité, masse spécifique, etc.

Les résultats analytiques obtenus déterminent l'épaisseur du film avec une bonne approximation, ce qui présente une importance particulière pour la conception de tout appareil utilisant ce procédé de graissage. Il existe, en effet, des

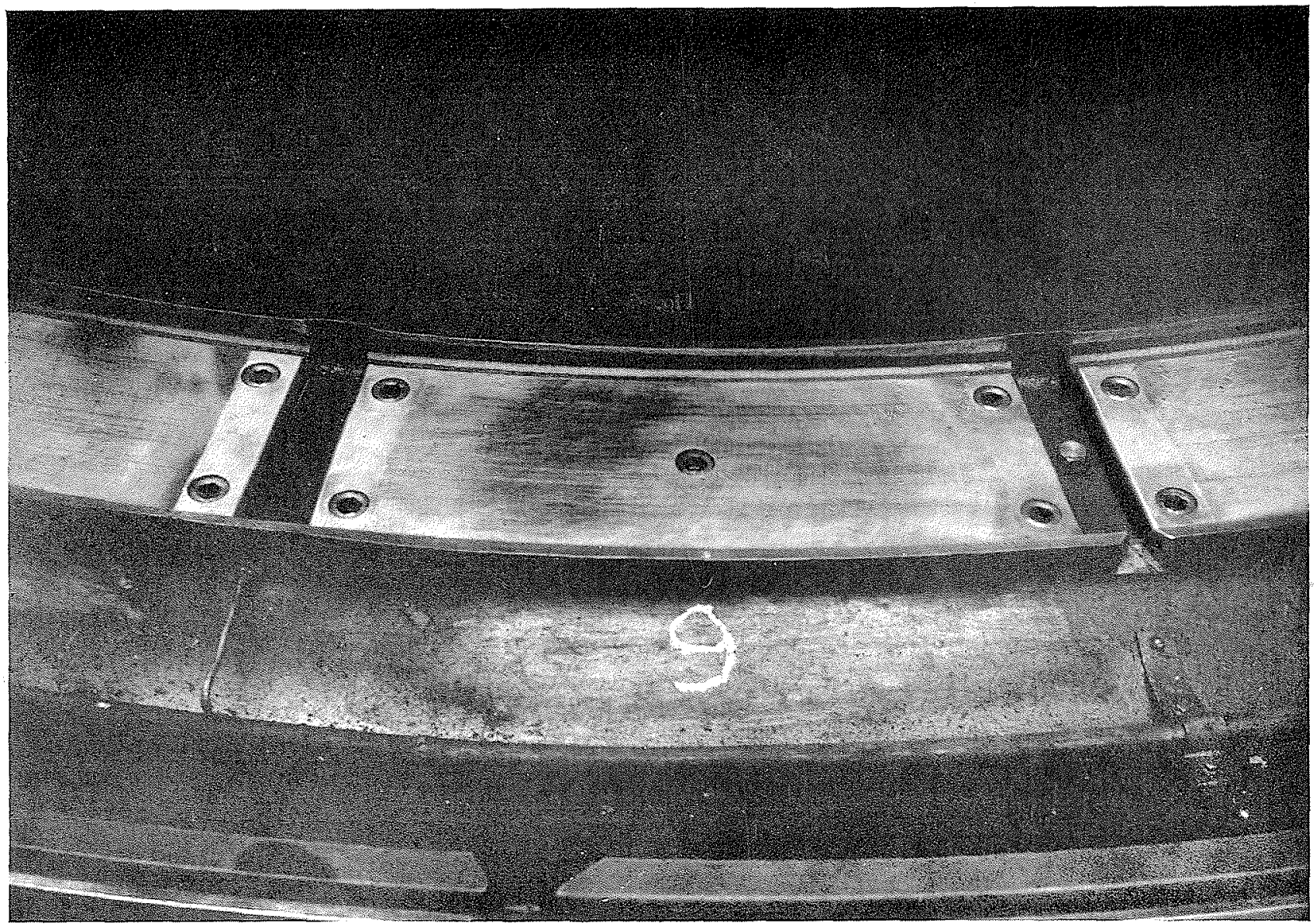

Fig. 6

Tour vertical de $6 \mathrm{~m}$. Photographie du portage du chemin de roulement plat du plateau de $6 \mathrm{~m}$. Lignes et surfaces de contact éclairage-électron 


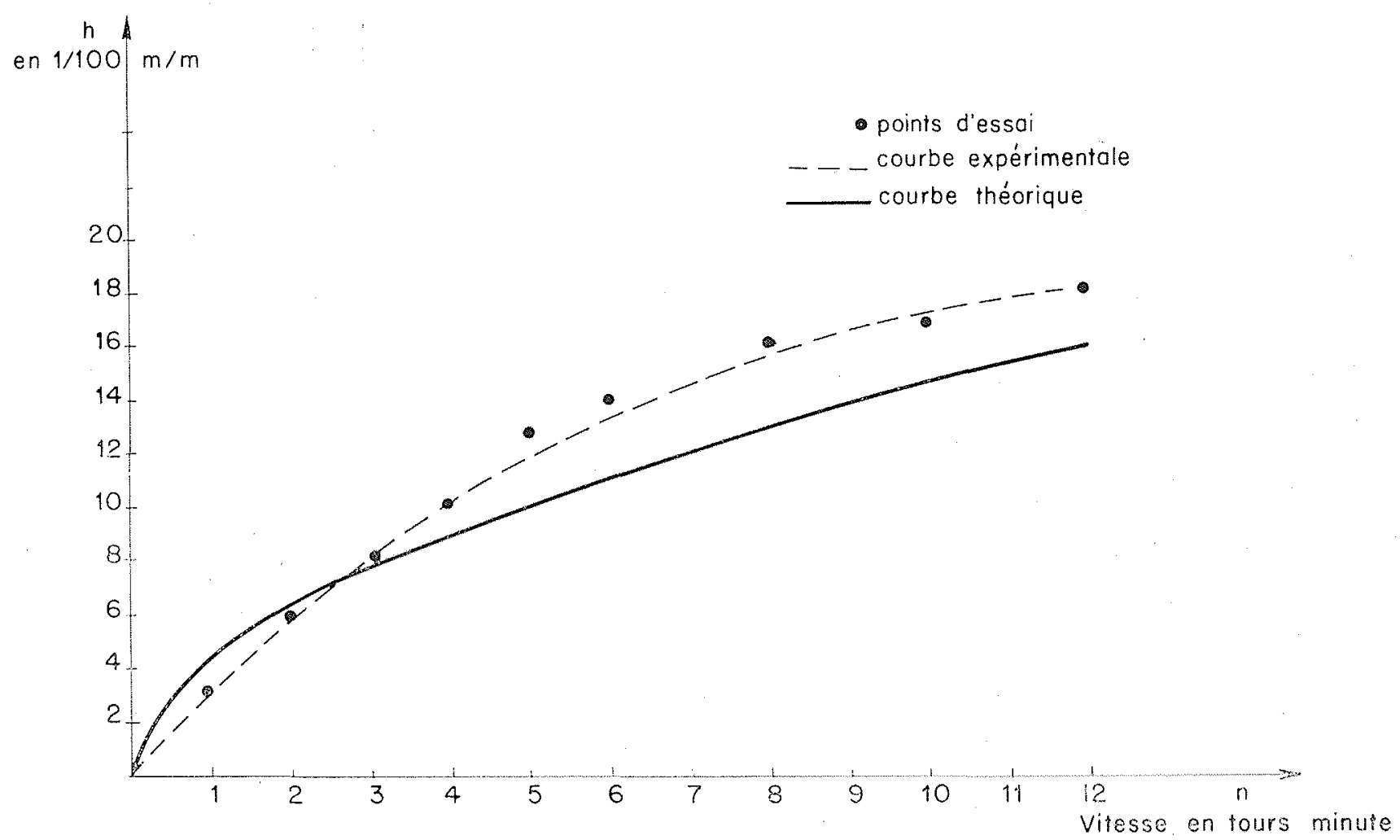

Fig. 7

machines (rectifieuses planes, tours verticaux), pour lesquelles la connaissance aussi exacte que possible de l'épaisseur du film d'huile est indispensable.

L'étude exposée précédemment permet aussi de comparer les domaines d'utilisation respectifs des pivoteries à grains parallèles et à patins basculants (type «MICHELL») de construction plus délicate. Nous aurons d'ailleurs l'occasion de revenir sur ce dernier type de pivoterie, une étude devant lui être consacrée dans un prochain numéro.

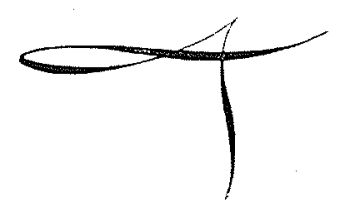

